\title{
Structural Basis for Degeneracy among Thermosensory Neurons in Caenorhabditis elegans
}

\author{
Xiang $\mathrm{Ma}^{1}$ and $\mathrm{Yu}$ Shen ${ }^{2}$ \\ ${ }^{1}$ Laboratory of Neurobiology and State Key Laboratory of Biomembrane and Membrane Biotechnology, College of Life Sciences, Peking University, Beijing \\ 100871, People's Republic of China, and 2Department of Organismic and Evolutionary Biology, Harvard University, Cambridge, Massachusetts 02138 \\ Review of Beverly et al.
}

Many biological systems show degeneracy, i.e., the ability to generate the same output by multiple distinct components under varying conditions. Degeneracy contributes to robustness by imparting a certain degree of redundancy into the network. It also increases adaptability by allowing flexible recruitment of different elements in a context-dependent manner. These properties of degeneracy are well embedded in the nervous system. A single neuron can project to several downstream targets, and inputs from multiple neurons often converge on the same integration site, where the final output is modulated. This convergent-divergent feature of neural connectivity provides an ideal substrate for functional degeneracy (Tononi et al., 1999).

The nematode Caenorhabditis elegans performs a robust navigational behavior called negative thermotaxis. When placed on a thermal gradient at temperatures higher than its cultivation temperature $\left(T_{\mathrm{C}}\right), C$. elegans migrates toward colder temperatures near $T_{\mathrm{C}}$. Early studies identified ciliated AFD neurons as primary thermosensory neurons; later studies unveiled the role of AWC sensory neurons in negative thermotaxis (Mori and Ohshima, 1995; Biron et al., 2008; Kuhara et al.,

Received Oct. 10, 2011; revised Nov. 2, 2011; accepted Nov. 2, 2011.

Correspondence should be addressed to Xiang Ma, College of Life

Sciences, Peking University, Beijing 100871, People's Republic of China. E-mail: xiangma.pku@gmail.com.

DOI:10.1523/JNEUROSCI.5112-11.2012

Copyright $\odot 2012$ the authors $\quad 0270-6474 / 12 / 320001-03 \$ 15.00 / 0$
2008). In a recent paper published in The Journal of Neuroscience, Beverly et al. (2011) identified the ASI chemosensory neuron as a new thermosensory neuron and showed that the three sensory neuron classes act degeneratively to generate a robust negative thermotaxis.

By genetic rescue and cell ablation experiments, the authors found that different combinations of AFD, AWC, and ASI neurons are sufficient or necessary for negative thermotaxis under various cultivation and assay temperatures, yet each responds to temperature stimuli uniquely in defined contexts. Particularly, ASI exhibited a stochastic $\mathrm{Ca}^{2+}$ transient in response to thermal stimuli within a $T_{\mathrm{C}^{-}}$ dependent operating range, distinct from both AFD and AWC neurons. The authors suggest that the robust negative thermotactic behavior of C. elegans relies on functional degeneracy and modulation among thermosensory neurons. The novel findings emphasize that well specified conditions are essential to such quantitative analysis of animal behavior and its cellular mechanisms.

An intriguing question, then, is how the newly identified ASI sensory neurons, together with other components, are engaged in a degenerate circuit underlying negative thermotaxis. One early model of thermotaxis comprises sensory neurons AFD and AWC, a layer of interneurons including AIY and AIZ, and a downstream command interneuron, RIA (Mori and Ohshima, 1995; Kuhara et al., 2008;
Garrity et al., 2010). In this model, thermotactic movement is regulated by two counterbalancing forces: AIY underlies a thermophilic drive that prompts migration toward higher temperatures, AIZ underlies a cryophilic drive for movement toward lower temperatures, and RIA balances these two drives guiding navigational behavior. How sensory information from ASI could be integrated into this hypothesized circuit to promote negative thermotaxis, as proposed by Beverly et al. (2011), is not clear. Although ASI neurons makes some synapses onto AIY and AIZ, the main synaptic output of ASI is on AIA, which also has few synaptic connections with AIZ and none with AIY (White et al., 1986). It is possible, however that ASI communicates with AIY or AIZ via neuroendocrine signals (Li and Kim, 2008) or synapses that have not been identified.

The seemingly attractive model of thermophilic/cryophilic drives described above was later challenged by multiple lines of evidence. On one hand, when examined in the context of navigational strategy, the roles of several key neurons involved in thermotaxis do not support their assumed functions in the two-drive model. The activity of sensory neurons AWC and AFD are positively correlated with animals' turning rates (Gray et al., 2005; Chalasani et al., 2007; Biron et al., 2008); Among the interneurons, AIA and AIY inhibit turns, AIB and AIZ promote turns, and ablation of RIA does not affect turning rates (Wakabayashi et al., 2004; 


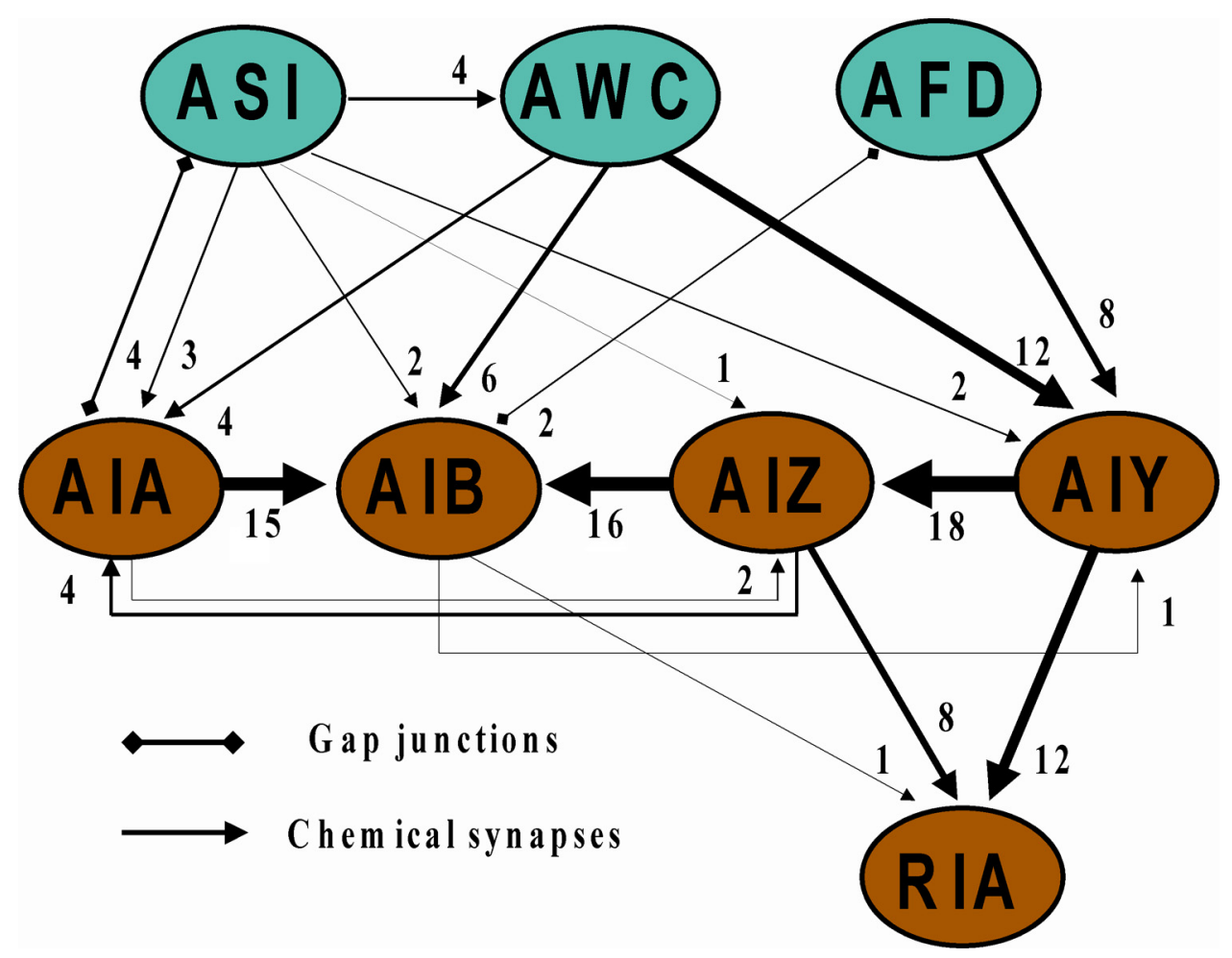

Figure 1. Synaptic connectivity between candidate sensory neurons (cyan ovals) and interneurons (orange ovals) in the hypothesized neural circuit for negative thermotaxis. The lines indicate synaptic connections between two neurons; the number of synapses is marked and the weights of lines are scaled to the number of synapses.

Gray et al., 2005). On the other hand, by analyzing responses to defined thermal gradient, Ryu and Samuel (2002) found no evidence for a thermophilic drive at temperatures below $T_{C}$; instead, their results suggested a biased random walk strategy for negative thermotaxis. C. elegans lengthens or shortens run duration in response to positive or negative temperature changes, respectively, when it moves through a thermal gradient at temperatures above $T_{\mathrm{C}}$. Thus, the overall navigation is biased toward $T_{\mathrm{C}}$ (Ryu and Samuel, 2002). A key point of this mechanism is the control of run duration (but not orientation) by turning events. To regulate turning, the thermosensory neurons may converge to a final common pathway, such as interneurons or downstream motoneurons that act as an integrator for sensorimotor transformation.

Which neuron(s) could serve as the locus of convergence in such a circuit? The synaptic connectivity of $C$. elegans nervous system provides us with some clues (Fig. 1). AIY is the major postsynaptic partner of AWC and AFD, and AIA is the main target of ASI outputs. Among the layer of interneurons, AIB receives heavy direct inputs from AIA and AIZ and indirect inputs from AIY via AIZ (White et al., 1986), which makes it a candidate for the final common pathway. Functionally, the
AIY-AIZ and AIA-AIB connections are likely to be inhibitory (Wakabayashi et al., 2004), whereas, based on behavioral analysis, the AIZ-AIB connection is likely to be excitatory (Wakabayashi et al., 2004; Gray et al., 2005). Considering the roles of these interneurons in modulating turning, we hypothesize that inputs from AFD, AWC, and ASI converge on the AIB interneuron via direct or indirect projections. How AIB may integrate sensory information in the degenerate circuit for negative thermotaxis and whether it is supported by current evidence are discussed below.

At temperatures higher than $T_{\mathrm{C}}$, all three sensory neurons are activated when they sense warming, except that ASI also senses cooling (Kimura et al., 2004; Biron et al., 2008; Kuhara et al., 2008; Beverly et al., 2011). As temperature rises, AFD may excite AIB through gap junctions (White et al., 1986), yet the excitation of AIB may not be strong enough to promote turning, which is consistent with the finding that AFD rescue alone in the tax- 4 mutants cannot restore negative thermotaxis (Beverly et al., 2011). Previous studies found that AWC excites AIB through AMPAtype glutamate receptors and inhibits AIY and AIA through glutamate-gated chloride channels or neuropeptide receptors (Chalasani et al., 2007, 2010); because the
AIY-AIZ and AIA-AIB connections are likely to be inhibitory (Wakabayashi et al., 2004), all three pathways could excite AIB neuron as the temperature increases. Although some activation of AWC promotes turning, the failure to restore negative thermotaxis by rescuing AWC alone in tax-4 mutants could be explained by the stochastic property of AWC's response to warming (Biron et al., 2008; Beverly et al., 2011). In contrast, the sum effect of AFD/AWC double rescue may provide sufficient excitation of AIB to execute normal thermotactic behavior (Beverly et al., 2011).

The inhibitory inputs of the newly identified ASI neurons onto AWC could contribute to the enhancement of negative thermotaxis when ASI was ablated in wild-type worms; indeed, this interpretation is supported by the restoration of normal navigational behavior with AWC/ ASI double ablation (Gray et al., 2005; Beverly et al., 2011). The property of ASIAIA connection is more complicated because of the existence of both chemical synapses and gap junctions (White et al., 1986). Given that the double rescue of ASI and AFD in tax-4 mutants results in normal navigational behavior (Beverly et al., 2011), it is likely that ASI inhibits AIA and excites AIB through disinhibition. Thus, ASI can affect turning frequency in at least 
two ways: inhibiting AWC neurons that promote turns and inhibiting AIA neurons that suppress turns. However, since ASI neurons sense both warming and cooling (Beverly et al., 2011), the net effect of ASI activation on turning frequency remains unclear and awaits further experiments.

A remaining question concerns the role of RIA neurons in negative thermotaxis. RIA receives synaptic inputs from both AIY and AIZ neurons (White et al., 1986). Ablation of AIY or AIZ results in cryrophilic or thermophilic phenotypes. Based on this, the earlier model proposed normal thermotaxis occurs when thermophilic and cryophilic drives mediated by AIY and AIZ are balanced (Mori and Ohshima, 1995). However, these effects are mainly observed when the worms are placed at their $T_{\mathrm{C}}$ rather than at higher temperatures. Considering that killing RIA or its main targets, RMD and SMD, did not change turning rates much (Gray et al., 2005), we speculate that RIA may integrate sensory information for isothermal tracking at temperatures near $T_{\mathrm{C}}$, when the hypothesized thermophilic and cryophilic drives serve in a weathervane strategy (Garrity et al., 2010). Ablation of AIY (or AIZ) terminates isothermal tracking and the remaining neuron AIZ (or AIY) biases the migration toward the colder (or warmer) direction. Whether ASI neurons are involved in isothermal tracking remains unclear, but it will not be too surprising if it is not, given the degeneracy among these thermosensory neurons.
The wiring diagram of C. elegans nervous system has been dissected at singleneuron resolution (White et al., 1986), facilitating the search for structural bases of functional degeneracy. By integrating the new findings of Beverly et al. (2011) with previous literature, we suggest that thermosensory signals from AFD, AWC, and ASI converge on the AIB interneuron in negative thermotaxis, whereas the AIYAIZ-RIA network may function mainly in isothermal tracking. The translocation of the putative integration locus to AIB fits the newly identified component ASI into a coherent circuit, which functions degenerately to execute a robust navigational strategy. We thus provide a testable hypothesis based on the most up-to-date collection of data for the structural basis of negative thermotaxis in C. elegans.

\section{References}

Beverly M, Anbil S, Sengupta P (2011) Degeneracy and neuromodulation among thermosensory neurons contribute to robust thermosensory behaviors in Caenorhabditis elegans. J Neurosci 31:11718-11727.

Biron D, Wasserman S, Thomas JH, Samuel AD, Sengupta P (2008) An olfactory neuron responds stochastically to temperature and modulates Caenorhabditis elegans thermotactic behavior. Proc Natl Acad Sci USA 105:11002-11007.

Chalasani SH, Chronis N, Tsunozaki M, Gray JM, Ramot D, Goodman MB, Bargmann CI (2007) Dissecting a circuit for olfactory behaviour in Caenorhabditis elegans. Nature 450:63-70.

Chalasani SH, Kato S, Albrecht DR, Nakagawa T, Abbott LF, Bargmann CI (2010) Neuropep- tide feedback modifies odor-evoked dynamics in Caenorhabditis elegans olfactory neurons. Nat Neurosci 13:615-621.

Garrity PA, Goodman MB, Samuel AD, Sengupta P (2010) Running hot and cold: behavioral strategies, neural circuits, and the molecular machinery for thermotaxis in C. elegans and Drosophila. Genes Dev 24:2365-2382.

Gray JM, Hill JJ, Bargmann CI (2005) A circuit for navigation in Caenorhabditis elegans. Proc Natl Acad Sci U S A 102:3184-3191.

Kimura KD, Miyawaki A, Matsumoto K, Mori I (2004) The C. elegans thermosensory neuron AFD responds to warming. Curr Biol 14: 1291-1295.

Kuhara A, Okumura M, Kimata T, Tanizawa Y, Takano R, Kimura KD, Inada H, Matsumoto K, Mori I (2008) Temperature sensing by an olfactory neuron in a circuit controlling behavior of C. elegans. Science 320:803-807.

Li C, Kim K (2008) Neuropeptides (September $25,2008)$, WormBook, ed. The C. elegans Research Community, WormBook, doi/10.1895/ wormbook.1.142.1, http://www.wormbook.org.

Mori I, Ohshima Y (1995) Neural regulation of thermotaxis in Caenorhabditis elegans. Nature 376:344-348.

Ryu WS, Samuel AD (2002) Thermotaxis in Caenorhabditis elegans analyzed by measuring responses to defined thermal stimuli. J Neurosci 22:5727-5733.

Tononi G, Sporns O, Edelman GM (1999) Measures of degeneracy and redundancy in biological networks. Proc Natl Acad Sci U S A 96:3257-3262.

Wakabayashi T, Kitagawa I, Shingai R (2004) Neurons regulating the duration of forward locomotion in Caenorhabditis elegans. Neurosci Res 50:103-111.

White JG, Southgate E, Thomson JN, Brenner S (1986) The structure of the nervous-system of the nematode Caenorhabditis elegans. Philos Trans R Soc B Biol Sci 314:1-340. 